\title{
Cardiac troponins and prediction of coronary artery disease risk
}

\author{
Anoop Dinesh Shah, PhD MRCP
}

\begin{abstract}
Cardiac troponins are released into the bloodstream as a result of myocardial injury and their detection in the blood is in routine clinical use for the diagnosis of myocardial infarction. A range of other cardiac and non-cardiac conditions may also cause a rise in troponin. A number of recent studies have found that high-sensitivity troponin is significantly associated with future coronary events in people without prior cardiovascular disease. Some studies have investigated its incremental benefit in risk prediction models, but have been too small to show a statistically significant improvement in the C statistic. Nevertheless, troponin measurements should be considered as a cardiovascular risk marker among healthy people as well as a diagnostic marker for myocardial infarction.
\end{abstract}


Cardiac troponins are proteins found in cardiac muscle cells, which can be released into the bloodstream as a result of myocardial injury. Their detection in the blood been used for over 15 years to aid the diagnosis of myocardial infarction. Troponin forms part of the troponintropomyosin complex that regulates cardiac muscle contraction, and consists of three subunits: troponin $\mathrm{T}$ which binds to tropomyosin, troponin $\mathrm{C}$ which binds calcium ions, and troponin I which is the inhibitory subunit. When the complex is activated by calcium ions, the tropomysin strands move to reveal myosin binding sites on the actin filaments, initiating muscle contraction.[1]

Assays for troponins I and $\mathrm{T}$ are in routine clinical use for measuring their concentration in the blood. Detection of a rise (and fall) in troponin is a key component of the current universal definition of myocardial infarction, and troponins are used clinically to differentiate acute coronary syndromes into unstable angina (no troponin release) and myocardial infarction (cardiac muscle damage due to ischaemia).[2] However, a range of other cardiac conditions (such as cardiomyopathies, arrhythmias and heart failure) and non-cardiac conditions (such as hypoxia, sepsis, pulmonary embolism, renal failure, stroke and strenuous exercise) may also cause a rise in troponin.[2] This means that troponin results always have to be interpreted in the context of the patient's history and other investigation results.

Healthy people have a very low concentration of troponin in the blood, and early (standard sensitivity) troponin assays were not precise enough to quantify this, but could allow a crude differentiation into 'detectable' versus 'non-detectable' troponin. Daniels et al. studied 5592 people without coronary disease in the Multi-Ethnic Study of Atherosclerosis, and found that male sex, diabetes, renal impairment, and higher NT-proBNP were associated with detectable troponin T.[3] There was also an association with ethnicity: African Americans were more likely to have detectable troponin T, whereas Chinese Americans were less likely. Study participants were followed for median 10.2 years and accrued 370 cardiovascular events (myocardial infarction, cardiac arrest or stroke). Individuals with detectable troponin $\mathrm{T}$ had a fourfold increased risk of coronary heart disease (95\% confidence interval (CI) 2.3, 6.8; $\mathrm{p}<0.001)$ and a threefold increased 
risk of cardiovascular disease (95\% CI 1.8, 4.8; p < 0.001) compared with individuals with undetectable troponin T.[3]

The study by Iribarren and colleagues [4] takes advantage of the quantification of low levels of troponin in healthy people that is possible using new high-sensitivity troponin assays. The study recruited 1135 people with no prior cardiovascular disease from the Kaiser Permanente health system in northern California, and followed them up for median 11.3 years. Baseline highsensitivity troponin I in the top quartile ( $>5.5 \mathrm{ng} / \mathrm{L}$ in men, $>4.2 \mathrm{ng} / \mathrm{L}$ in women) was associated with increased risk of coronary heart disease compared to the bottom quartile, even after adjustment for age, sex, race, ethnicity, education level, diabetes, C-reactive protein, renal function (eGFR) and the Framingham risk score (hazard ratio 2.47, 95\% CI 1.55, 3.93).[4]

These findings are consistent with those from other studies. In the AGES-Reykjavik study of 5764 older Icelandic individuals (age 66-98 years), incidence of all-cause mortality, cardiovascular disease or coronary heart disease was significantly associated with increasing concentrations of troponin I.[5] In the Caerphilly study, Patterson et al. compared the strength of association of cardiac troponin concentrations with cardiovascular and non-cardiovascular mortality. They followed up 1773 Welsh men (667 with prior cardiovascular disease) for mean 15.4 years and found that higher troponin was significantly associated with cardiovascular death (subhazard ratio 1.71 per tertile increase, 95\% CI 1.46, 1.99) but not non-cardiovascular death (subhazard ratio 1.00, 95\% CI 0.89, 1.11; p for interaction $<0.001)$.[6]

It is biologically implausible that circulating troponins cause increased risk of coronary disease; instead they may reflect the severity of the underlying atherosclerosis or subclinical myocardial ischaemia, or may be proxies for conditions associated with increased cardiovascular risk such as renal impairment. However, the exact biological mechanism underlying these findings is not clearly understood.

Iribarren et al. also sought to investigate the utility of high sensitivity troponin I measurements in cardiovascular risk prediction. Their sample size with 164 coronary events was too small so show a 
statistically significant increment in the $\mathrm{C}$ statistic, but the category-based net reclassification index was 18\% (95\% CI 8\%, 30\%).[4] Similarly, Welsh et al. found a non-significant improvement in classification when troponin T measurements were included in QRISK2 or ASSIGN cardiovascular risk prediction models applied to the British Regional Heart Study (3538 men) and MIDSPAN Family Study (1907 men and women) [7]. Further studies with larger sample sizes would be required to fully evaluate high sensitivity troponin in cardiovascular risk prediction among healthy people before it could be recommended for clinical use.

Investigating the prognostic significance of troponins in the general population may also help to inform the diagnosis of myocardial infarction, which relies on the interpretation of 'abnormal' troponin results, defined as greater than the $99^{\text {th }}$ percentile upper reference limit. The determination of such reference ranges can be strongly influenced by the composition of the reference population. [8] Longitudinal cohort studies may enable normality to be defined as the group with good prognosis, rather than individuals considered 'normal' based on prior history and current clinical features. Interpretation of troponin results using age and sex-specific reference ranges may help to avoid under- or over-diagnosis of myocardial infarction.

In conclusion, blood concentrations of cardiac troponins convey prognostic information in people without cardiovascular disease as well as those with acute coronary syndromes. This may herald a paradigm shift, with future use of troponin measurements for the evaluation of stable patients in primary care, as well as patients with acute coronary syndrome. However, studies to date have been too small to evaluate whether any improvement in decision making as a result of including troponin in cardiovascular risk models improves outcomes or is cost-effective. Larger population-based cohort studies, with comprehensive risk factor and biomarker measurement, would be required to fully assess the clinical utility of troponins in cardiovascular risk prediction. 


\section{References}

1. Takeda S, Yamashita A, Maeda et al. Structure of the core domain of human cardiac troponin in the Ca2+-saturated form. Nature 2003;424:35-41. doi:10.1038/nature01780

2. Thygesen K, Alpert JS, Jaffe AS, et al. Third universal definition of myocardial infarction. Circulation 2012;126(16):2020-35. doi: 10.1161/CIR.0b013e31826e1058.

3. Daniels LB, Clopton P, deFilippi CR, et al. Serial measurement of N-terminal pro-B-type natriuretic peptide and cardiac troponin $\mathrm{T}$ for cardiovascular disease risk assessment in the Multi-Ethnic Study of Atherosclerosis (MESA). Am Heart J 2015;170(6):1170-83. doi: 10.1016/j.ahj.2015.09.010.

4. Iribarren C, Chandra M, Rana J, et al. High-sensitive Cardiac Troponin-I and Incident Coronary Heart Disease among Asymptomatic Older Adults. Heart 2016.

5. Thorsteinsdottir I, Aspelund T, Gudmundsson E, et al. High-Sensitivity Cardiac Troponin I is a Strong Predictor of Cardiovascular Events and Mortality in the AGES-Reykjavik Community Based Cohort of Older Individuals. Clin Chem 2016. doi: 10.1373/clinchem.2015.250811.

6. Patterson CC, Blankenberg S, Ben-Shlomo Y, et al. Which biomarkers are predictive specifically for cardiovascular or for non-cardiovascular mortality in men? Evidence from the Caerphilly Prospective Study (CaPS). Int J Cardiol 2015; 201: 113-118. doi: 10.1016/j.ijcard.2015.07.106

7. Welsh P, Hart C, Papacosta O, et al. Prediction of Cardiovascular Disease Risk by Cardiac Biomarkers in 2 United Kingdom Cohort Studies Does Utility Depend on Risk Thresholds For Treatment? Hypertension 2016;67:309-315 doi:

10.1161/HYPERTENSIONAHA.115.06501

8. Collinson PO, Heung YM, Gaze D, et al. Influence of population selection on the 99th 
percentile reference value for cardiac troponin assays. Clin Chem 2012;58(1):219-25. doi: 10.1373/clinchem.2011.171082. 